\title{
Microstructures and Recorded Magnetization Microstructures of Co-based Thin Film Recording Media
}

\author{
Masaaki FUTAMOTO and Yukio HONDA \\ Central Research Laboratory, Hitachi Ltd. \\ Kokubunji Tokyo 185, Japan
}

\begin{abstract}
Inter-relationships among medium microstructure, magnetization structure, and recording characteristics are investigated for Co-based longitudinal, perpendicular, and obliquely evaporated recording media. Two types of magnetization irregularities are observed. One is the magnetization transition irregularity which causes magnetic interference between neighboring recorded bits at high linear recording densities. The magnetization interference is the main source of the medium noise for longitudinal recording media. The second type of the magnetization irregularity exists inside the recorded bits which can be observed under all types of recording media. The size of the magnetization irregularity is several times greater than the average size of magnetic crystal grain. The size of the latter magnetization irregularity should be decreased well below the shortest bit length in order to decrease the medium noise and to improve the linear recording density characteristics.
\end{abstract}

\section{INTRODUCTION}

Medium noise reduction is very important to increase the areal density of magnetic recording medium, especially when the medium is combined with use of a high sensitivity magnetoresistive(MR) head. To reduce the medium noise, the magnetic coupling between the crystal grains, which make up a recording medium, has to be decreased. The coupling can be decreased either by separating the crystal grains physically or by separating magnetically with enhanced segregation of nonmagnetic elements at the grain boundaries[1-3]. The effectiveness of increasing the $\mathrm{Cr}$ concentration to reduce the medium noise for Co-based $\mathrm{CoCrTa}[4]$ and $\mathrm{CoCrPt}[5,6]$ longitudinal recording media has been shown. The feasibility of $1-2 \mathrm{~Gb} / \mathrm{in}^{2}$ storage densities has been demonstrated with longitudinal magnetic recording under non-contact conditions $[7,8]$. With contact recording, very high linear recording densities greater than $300 \mathrm{kFCI}[9,10]$ and high track densities greater than $17 \mathrm{kTPI}$ have been shown with perpendicular magnetic recording[11,12].

As the areal density increases, the magnetic unit cell size approaches the size of magnetic crystal grain which make up a recording medium. For example, at $10 \mathrm{~Gb} / \mathrm{in}^{2}$ areal density, the magnetic unit cell size is only $0.06 \mu \mathrm{m}^{2}$ including less than one hundred crystal grains. Thus, investigating the relationship between thin film structure and recording characteristics is crucial in order to effectively improve the recording medium microstructure which is suitable for high density magnetic recording.

In this research, we investigate the relationship between recording medium microstructure and recorded magnetization microstructure for longitudinal CoCrPt/CoCrPtSi, CoCrTa media, a perpendicular $\mathrm{CoCrTa}$ medium, and an obliquely evaporated $\mathrm{Co}-\mathrm{CoO}$ thin film medium. We will clarify the difference in recorded magnetization structures among these recording media. Possibilities of improving the media microstructures that decrease the media noise even further will also be discussed.

\section{MEDIA STRUCTURES AND MAGNETIC PROPERTIES}

The media structure, the basic magnetic properties, and some of the recording characteristics of Co-based high density recording media are summarized in Table 1 . The specifications of magnetic heads employed to record and to reproduce signals are also given in this Table. The $\mathrm{CoCr}_{5} \mathrm{Pt}_{21} / \mathrm{CoCr}_{14} \mathrm{Pt}_{8} \mathrm{Si}_{3}$ longitudinal recording medium was formed on a plain glass substrate with $\mathrm{Cr}$ underlayer[3]. A high $\mathrm{Ar}$ pressure was applied to produce a physically isolated grain structure to reduce the medium noise. The preferred orientation of the $\mathrm{Cr}$

Table 1 Structures and magnetic properties of Co-based thin film recording media

\begin{tabular}{|c|c|c|c|c|c|c|c|c|}
\hline & \multicolumn{3}{|c|}{ LONGITUDNAL RECORDING MEDIA } & \multirow{2}{*}{\multicolumn{2}{|c|}{$\begin{array}{c}\text { PERPENDICULAR RECORDING MEDIUM } \\
\text { CoCr17Ta5 } \\
\end{array}$}} & \multirow{2}{*}{\multicolumn{2}{|c|}{$\begin{array}{c}\text { OBLIQUELY EVAPORATED MEDIUM } \\
\text { CO-COO } \\
\end{array}$}} \\
\hline & & $\mathrm{CoCr} 5 \mathrm{P} 21 / \mathrm{CoCr} 4 \mathrm{P} 21 \mathrm{Si3}$ & \multirow{2}{*}{ 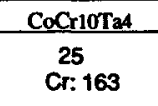 } & $\mathrm{CoCr} 16 \mathrm{Ta4}$ & & & & \\
\hline 焉 & $\begin{array}{l}\text { Magnetic Layer (nm) } \\
\text { Underiayer (nm) } \\
\text { Substrate }\end{array}$ & $\begin{array}{l}15+15 \\
\text { Cr: } 150 \\
\text { Glass }\end{array}$ & & $\begin{array}{l}31 \\
\text { Cr: } 150 \\
\text { NiP/Al }\end{array}$ & \multicolumn{2}{|c|}{$\begin{array}{c}100 \\
\text { Ti:(20nm)/TiC } 10(30 \mathrm{~nm})\end{array}$} & \multicolumn{2}{|c|}{$\begin{array}{c}200 \\
\text { None } \\
\text { Polyechylene Terephthalate }\end{array}$} \\
\hline 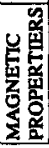 & $\begin{array}{l}\text { Mrt (G } \mu \mathrm{m}) \\
\mathrm{Hc}(\mathrm{l})(\mathrm{kOe}) \\
\mathrm{Hc}(\mathrm{l}) / \mathrm{Hc}(\mathrm{r})\end{array}$ & $\begin{array}{l}165 \\
2.3 \\
1.0\end{array}$ & $\begin{array}{l}154 \\
1.93 \\
1.41\end{array}$ & $\begin{array}{r}168 \\
1.90 \\
1.59\end{array}$ & $\begin{array}{c}\text { Ms } \\
\mathrm{Mr} / \mathrm{Ms} \\
\mathrm{Hc}\end{array}$ & $\begin{array}{l}440 \mathrm{emu} / \mathrm{cc} \\
0.34 \\
1.48 \mathrm{kOe}\end{array}$ & $\begin{array}{c}\text { Ms } \\
\mathrm{Mr} / \mathrm{Ms} \\
\mathrm{Hc}\end{array}$ & $\begin{array}{l}850 \mathrm{emu} / \mathrm{cc} \\
0.37 \\
1.06 \mathrm{kOe}\end{array}$ \\
\hline \multicolumn{2}{|c|}{ - 6dB Linear Density D50 (kFCI) } & 80 & 86 & 87 & \multicolumn{2}{|r|}{83} & & 120 \\
\hline 高 & $\begin{array}{l}\text { Write Head } \\
\text { Read Head }\end{array}$ & 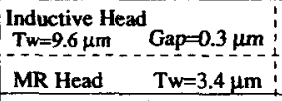 & $\begin{array}{l}\text { Inductive Head } \\
\text { Tw=10 } 10 \mathrm{~m} \\
\text { MR Head }\end{array}$ & $\begin{array}{l}\text { Gap }=0.4 \mu \mathrm{m} \\
\mathrm{Tw}=3.6 \mu \mathrm{m}\end{array}$ & \multicolumn{2}{|c|}{$\begin{array}{l}\text { Inductive Head } \\
\text { Tw=10.5 } \mu \mathrm{m} \\
\text { MR Head }\end{array}$} & \multicolumn{2}{|c|}{$\begin{array}{l}\text { MIG Head } \\
\mathrm{Tw}=26 \mu \mathrm{m} \mathrm{Gap}=0.27 \mu \mathrm{m}\end{array}$} \\
\hline \multicolumn{2}{|c|}{ Magnetic Spacing (nm) } & 80 & \multicolumn{2}{|c|}{120} & \multicolumn{2}{|r|}{90} & \multicolumn{2}{|c|}{ Contact Recording } \\
\hline
\end{tabular}

$\mathrm{Hc}(\mathrm{l}) / \mathrm{Hc}(\mathrm{r})$ : Coercivity anisotropy measured along the circumferential( $\mathrm{Hc}(\mathrm{l}))$ and radial( $\mathrm{Hc}(\mathrm{r}))$ directions of disk substrate.

Magnetic properties are measured using VSM by applying a magnetic field parallel to the easy magnetization directions of the specimens. 
underlayer is (110). The epitaxial growth plane of magnetic layer is (1011) where the easy magnetization axes of magnetic crystals are 28 degrees off from the film plane. This is a magnetically isotropic medium. On the contrary, the CoCrTa media[4] were formed on textured $\mathrm{NiP} / \mathrm{Al}$ substrates after depositing $\mathrm{Cr}$ underlayers employing a low Ar pressure to yield continuous film structures. The $\mathrm{Cr}$ concentration in magnetic films was varied from $\mathrm{CoCr}_{10} \mathrm{Ta}_{4}$ to $\mathrm{CoCr}_{16} \mathrm{Ta}_{4}$ to investigate the effect of $\mathrm{Cr}$ composition on the magnetization microstructures. The preferred orientation of the $\mathrm{Cr}$ underlayer was (100). The (1120) plane of magnetic layer was epitaxially grown on the $(100)$ oriented $\mathrm{Cr}$ underlayer. The easy magnetization axes of these media lied in-plane. These media have coercivity anisotropies when measured along the circumferential and the radial directions of the disk substrates. The single layer perpendicular recording medium of $\mathrm{CoCr}_{17}$ Tas was prepared on an oxidized silicon substrate employing a $\mathrm{Ti} / \mathrm{TiCr}_{10}$ underlayer to enhance the growth of c-axis oriented columns[13]. The read/write characteristics of these media were measured using

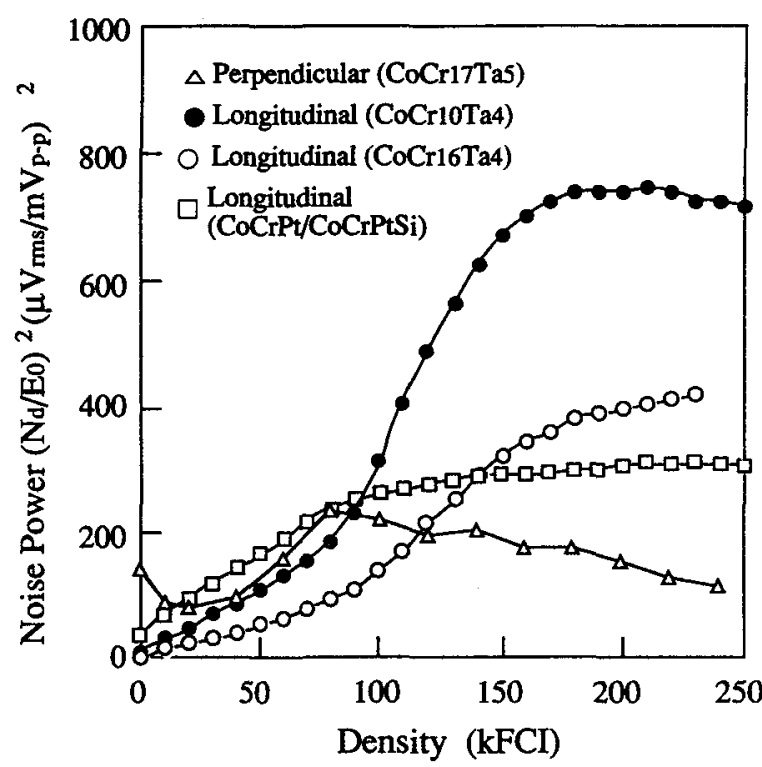

Fig.1 Dependences of media noise on recording density for $\mathrm{CoCrPt} / \mathrm{CoCrPtSi}$, CoCrTa longitudinal media and for $\mathrm{CoCrTa}$ perpendicular recording medium.

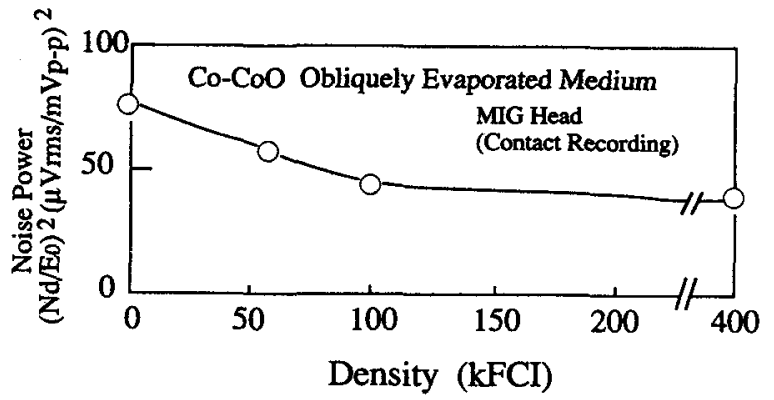

Fig. 2 Dependence of medium noise on recording density for Co-CoO obliquely evaporated thin film. inductive thin film heads for writing and MR heads for reproducing recorded signals and for evaluating media noise. The track width of MR heads was kept nearly constant at $3.4-3.6 \mu \mathrm{m}$.

The $\mathrm{Co}-\mathrm{CoO}$ thin film medium was prepared using an oblique vacuum evaporation of Co on a polyethylene terephthalate substrate at room temperature in an oxygen atmosphere[14-16]. The basic magnetic properties of Co$\mathrm{CoO}$ medium were measured using a vibrating sample magnetometer by applying a magnetic field perpendicularly to the easy magnetization axis of the medium. The easy magnetization axis was 60 degrees from the substrate surface. A metal-in-gap(MIG) head was used in the contact mode with a head-to-medium relative velocity of $2.5 \mathrm{~m} / \mathrm{s}$ for the read/write experiment of the $\mathrm{Co}-\mathrm{CoO}$ medium.

The variations in the media noise when the linear recording density is increased are shown in Figs. 1 and 2 for these media. The noise of the longitudinal recording media, CoCrPt/CoCrPtSi and CoCrTa, increases as the recording density increases, while those of perpendicular and obliquely evaporated media remain almost constant or even decrease. The noise levels are mostly determined by the noise at $\mathrm{dc}$ erased states for the perpendicular and obliquely evaporated recording media. However, the noise of CoCrTa longitudinal recording media begins to increase sharply at densities greater than $100 \mathrm{kFCI}$.
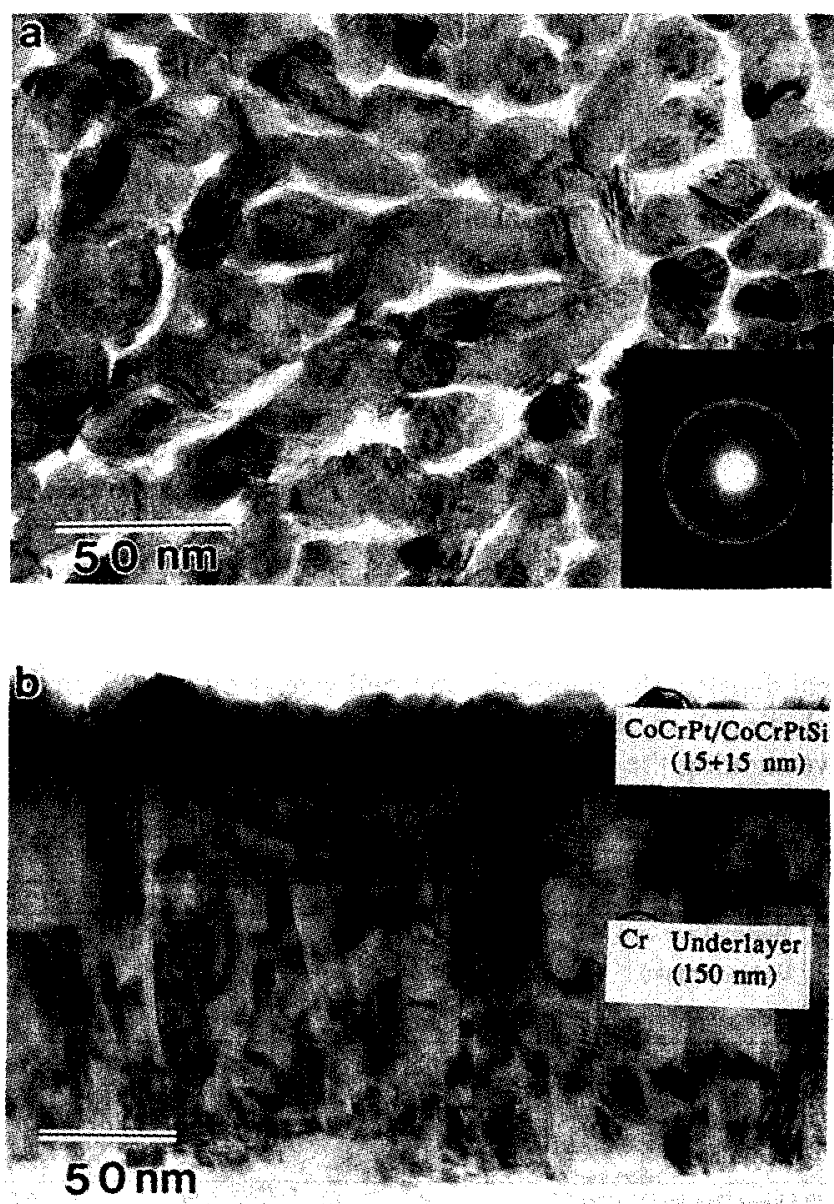

Fig.3 (a)Plane-view and (b)cross-sectional transmission electron micrographs of $\mathrm{CoCrPt} / \mathrm{CoCrPtSi}$ longitudinal recording medium. 


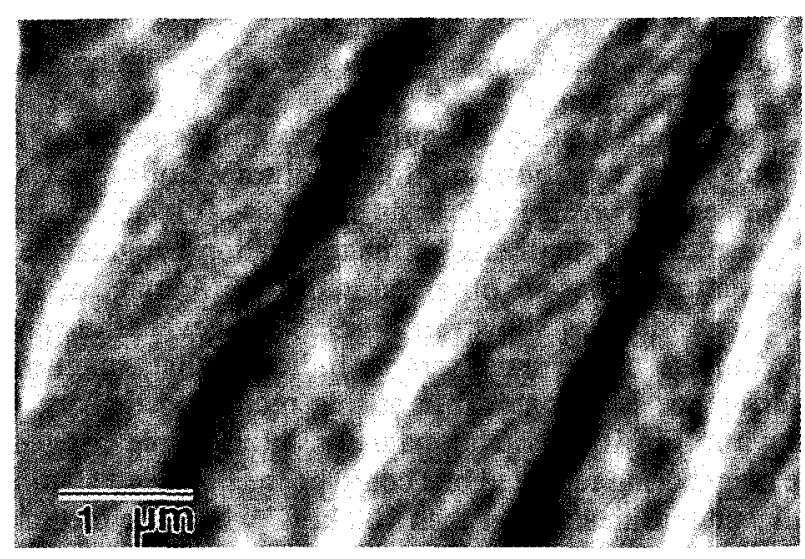

Fig.4 Magnetic force microscope image of $\mathrm{CoCrPt} / \mathrm{CoCrPtSi}$ medium recorded at $20 \mathrm{kFCI}$.

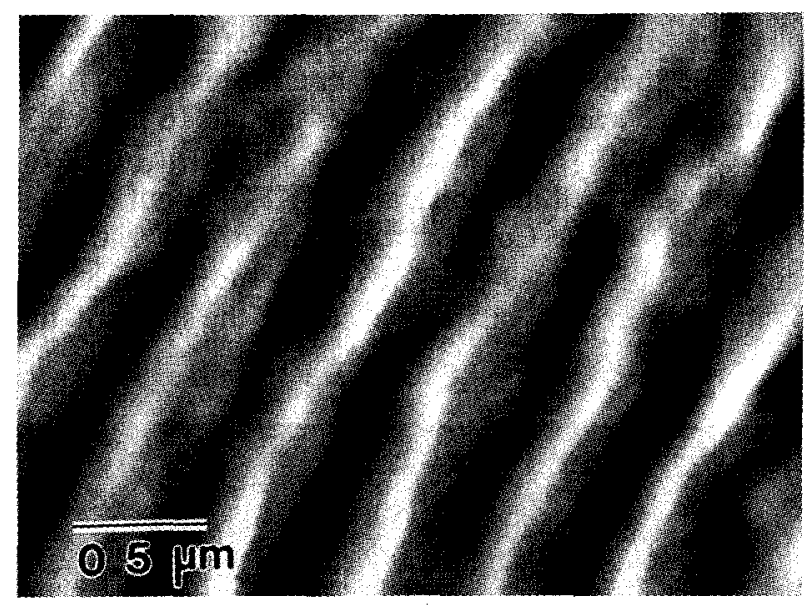

Fig.5 Magnetic force microscope image of $\mathrm{CoCrPt} / \mathrm{CoCrPtSi}$ medium recorded at $100 \mathrm{kFCl}$.

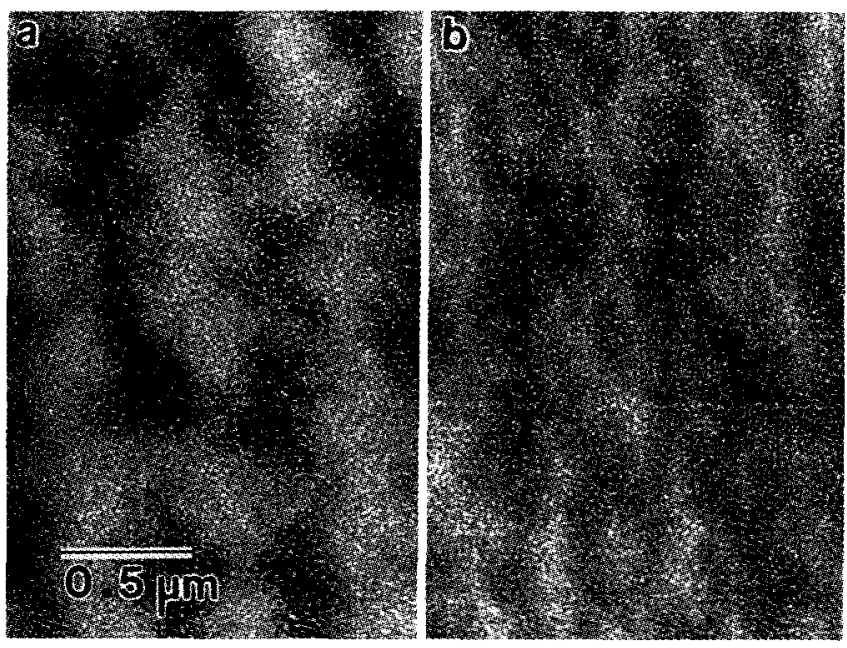

Fig.6 Spin-SEM images of $\mathrm{CoCrPt} / \mathrm{CoCrPtSi}$ medium recorded at $100 \mathrm{kFCI}$. (a)Horizontal and (b)vertical magnetization component images.
The differences in noise behavior between these media are probably correlated to the differences in the recorded magnetization microstructures of the respective specimens.

\section{LONGITUDINAL RECORDING MEDIA}

\subsection{Physically Grain Separated CoCrPt/CoCrPtSi Longitudinal Recording Medium}

Plane-view and cross-sectional transmission electron micrographs(TEM) of physically grain separated $\mathrm{CoCrPt} / \mathrm{CoCrPtSi}$ medium are shown in Fig.3. The magnetic crystal grains, whose size ranges from $20-30$ $\mathrm{nm}$, form chain-like clusters consisted of several grains[17]. Neighboring clusters are physically separated with an average spacing of $2 \mathrm{~nm}$. Electron diffraction data shows that the crystalline easy axes of the grains are oriented randomly on the film plane.

The magnetic force microscope(MFM) image of the sample recorded at $20 \mathrm{kFCI}$, Fig.4, shows bright and dark parallel lines which correspond to the bit transitions. There are contrast variations with dimensions of $0.1-0.5$ $\mu \mathrm{m}$ between the bit transitions. Comparison of an atomic force gradient image with an MFM image obtained from a same area indicated that these MFM contrast variations correspond to magnetic clusters which consist of several magnetically-coupled crystal grains[18]. Similar magnetization irregularities were observed in a dc erased area. Such irregularities must be related to the dc erased noise, since the MFM image reflects the stray magnetic field distributions very close to the recorded sample surface which a magnetic head senses as an accumulation along the effective track width when reproducing the recorded signals. As the bit length approaches the size of magnetization irregularities, the shape of the magnetization transition becomes distorted with curvatures corresponding to the size of the magnetization irregularities and becomes even discontinuous as shown in the MFM image of the 100 kFCI recording(Fig.5). The magnetic interference between the neighboring recorded bits became noticeable at 100 $\mathrm{kFCl}$ as shown more clearly in the surface magnetization image observed by spin-SEM[19]. Fig.6 shows a pair of spin-SEM images of a sample recorded at $100 \mathrm{kFCI}$ for horizontal( $x)$ and vertical( $y)$ magnetization components[20]. The horizontal and vertical directions are parallel to the circumferential and radial directions of disk media, respectively. The transition width is estimated to be around $0.1-0.2 \mu \mathrm{m}$. Bit cross-talks are clearly observed at this recording density. Furthermore, the spinSEM image shows that the size of magnetization irregularities is $0.1-0.3 \mu \mathrm{m}$ which corresponds to the fluctuations of leakage magnetization fields observed by MFM. The round shape of the magnetization irregularities observed in the horizontal magnetization component image shown in Fig.6(a) probably reflects the isotropic magnetic properties of this specimen. It is necessary, therefore, to decrease the size of magnetization irregularities in order to further decrease the medium noise and to improve the linear recording density characteristics. 


\subsection{CoCrTa Longitudinal Recording Media.}

The medium noise could be decreased without employing a physically separated grain structures by increasing the $\mathrm{Cr}$ content in the CoCrTa media [4]. In the case of $\mathrm{CoCr}_{\mathbf{x}} \mathrm{Ta}_{4}$ media, the medium noise decreased about $50 \%$ as the $\mathrm{Cr}$ content( $\mathrm{x}$ ) increased from 10 to 16 at\%. The film microstructures of $\mathrm{CoCr}_{10} \mathrm{Ta}_{4}$ and $\mathrm{CoCr}_{16} \mathrm{Ta}_{4}$ media were, however, very similar. This is because the size and the crystallographic orientation of magnetic crystals are determined by the epitaxial growth of Co-based alloy on the $\mathrm{Cr}$ underlayers. A plane-view

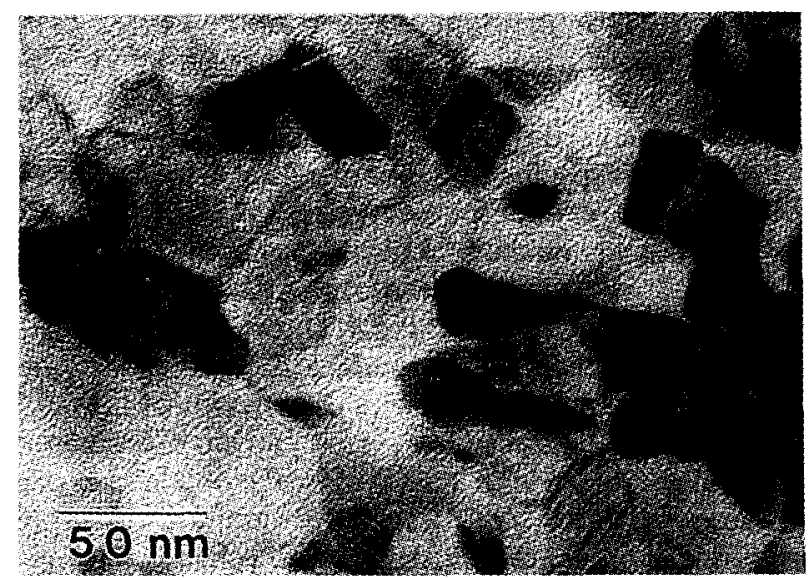

Fig.7 Plane-view TEM micrograph of $\mathrm{CoCr}_{16} \mathrm{Ta}_{4}$ longitudinal recording medium.

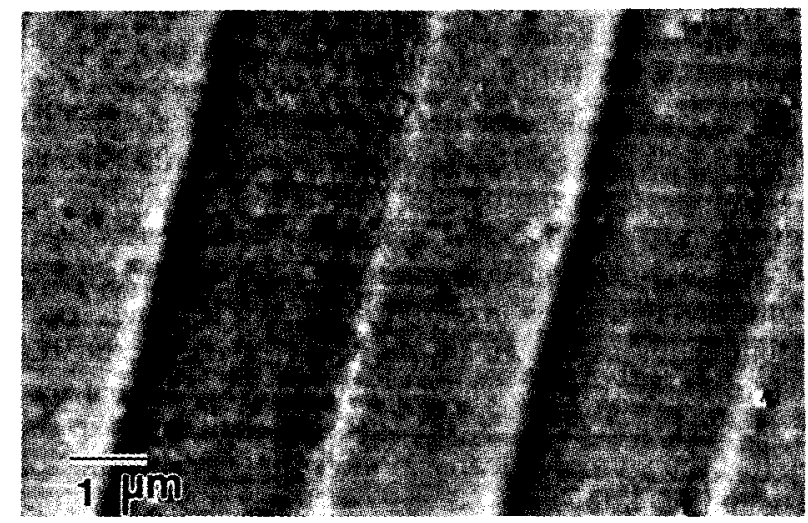

Fig.8 Magnetic force microscope image of $\mathrm{CoCr}_{16} \mathrm{Ta}_{4}$ medium recorded at $10 \mathrm{kFCI}$.

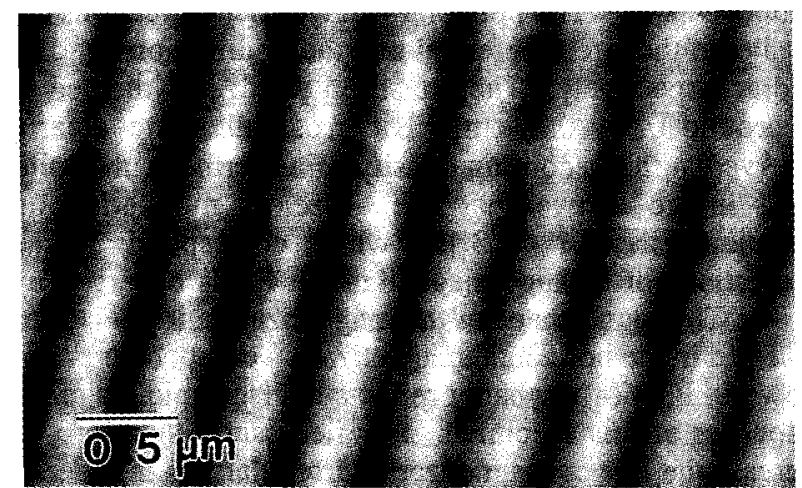

Fig.9 Magnetic force microscope image of $\mathrm{CoCr}_{16} \mathrm{Ta}_{4}$ medium recorded at $100 \mathrm{kFCI}$.
TEM micrograph of a $\mathrm{CoCr}_{16} \mathrm{Ta}_{4}$ medium is shown in Fig.7. The magnetic crystal grains, whose average diameter is about $20 \mathrm{~nm}$, are in close contact with no clear physical separation.

The magnetization irregularities within the range of recorded bit transitions of this medium as observed by MFM as shown in Fig. 8 is as small as $0.1-0.15 \mu \mathrm{m}$. Because of this small size of magnetization irregularities, clear bit transitions are observed in the MFM image of 100 kFCI recording as shown in Fig.9. However, the spinSEM image of the same sample indicates that magnetic interference is recognizable at this recording density as shown in Fig.10(a). The magnetic interference between the neighboring recorded bits could be be observed starting at linear densities greater than $100 \mathrm{kFCI}$ where the medium noise begins to increase sharply[21]. At a higher recording density of $140 \mathrm{kFCl}$, the incidence of magnetic interference, or bit cross-talk, increases as shown in Fig.10(b). The average size of magnetization irregularities observed by spin-SEM is $0.12 \mu \mathrm{m} \times 0.20 \mu \mathrm{m}$, elongated along the circumferential direction of the disk. The anisotropic shape is due to the magnetic anisotropy of the medium. The average size increases up to $0.16 \mu \mathrm{m} \times 0.30$ $\mu \mathrm{m}$ for the $\mathrm{CoCr}_{10} \mathrm{Ta}_{4}$ medium, as shown in Fig.11(a), which shows a medium noise at $100 \mathrm{KFCl}$ about twice as large as that of the $\mathrm{CoCr}_{16} \mathrm{Ta}_{4}$ medium. For $140 \mathrm{kFCI}$ recording, bit cross-talks extend more than two neighboring recorded bits which causes the shape of the
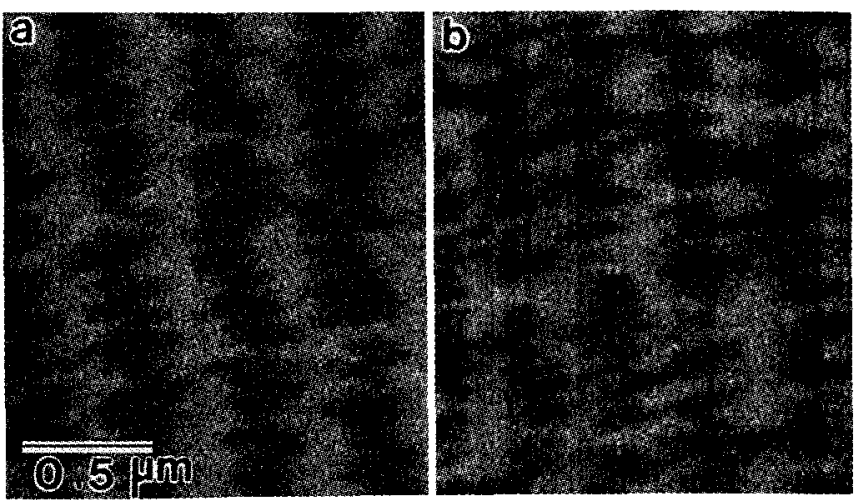

Fig.10 Spin-SEM images of $\mathrm{CoCr}_{16} \mathrm{Ta}_{4}$ sample recorded at (a) $100 \mathrm{kFCI}$ and (b) $140 \mathrm{kFCI}$.
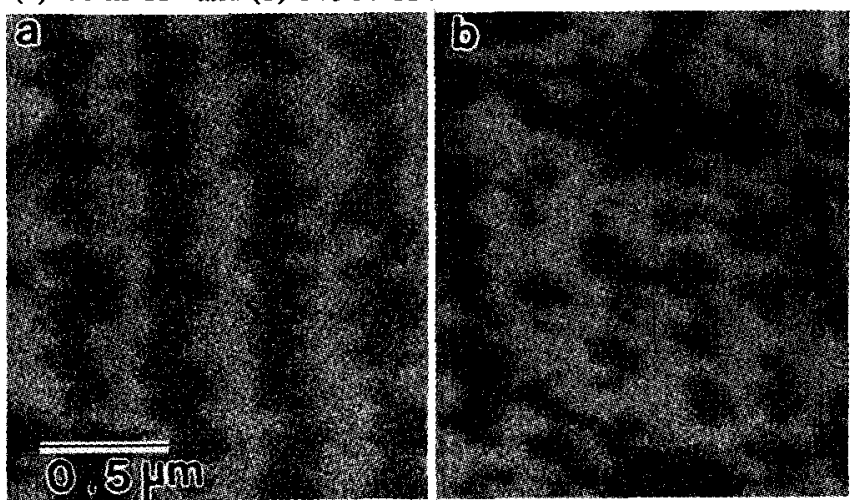

Fig.11 Spin-SEM images of $\mathrm{CoCr}_{10} \mathrm{Ta}_{4}$ sample recorded at (a) $100 \mathrm{kFCI}$ and (b) $140 \mathrm{kFCI}$. 
magnetization transitions to deform as shown in Fig.11(b). The difference in the magnetization irregularities is attributed to $\mathrm{Cr}$ segregation to the grain boundaries. The $\mathrm{Cr}$ concentration at grain boundaries was greater for the Cr-enriched $\mathrm{CoCr}_{16} \mathrm{Ta}_{4}$ sample. For the $\mathrm{CoCr}_{16} \mathrm{Ta}_{4}$ sample, the $\mathrm{Cr}$ concentration at grain boundaries was determined to be greater than 25 at\% and the thickness of Cr-enriched region was estimated to be $1-2 \mathrm{~nm}$ by electron micro probe analysis using a high resolution transmission electron microscope[22]. The presence of the Cr-enriched region around the magnetic crystals plays an important role in decreasing the magnetic interaction between magnetic crystal grains thus decreasing the size of magnetization irregularities which is closely related to the reduction in medium noise.

\section{PERPENDICULAR RECORDING MEDIUM}

It was possible to increase the linear recording density with a $\mathrm{CoCr}_{17} \mathrm{Ta}_{5}$ perpendicular recording medium by combining an inductive thin film head for writing and an MR head for reading[13]. At linear densities greater than $150 \mathrm{kFCI}$, higher signal to noise ratio was confirmed with the $\mathrm{CoCr}_{17} \mathrm{Ta}_{5}$ single layer medium than with the $\mathrm{Cr}$ enriched $\mathrm{CoCr}_{17} \mathrm{Ta}_{5}$ longitudinal recording medium evaluated under a similar condition. High resolution SEM and cross-sectional TEM images of the film structure for the perpendicular recording medium are shown in Fig.12. The medium consists of c-axis oriented columnar crystals whose average diameter is about $35 \mathrm{~nm}$.
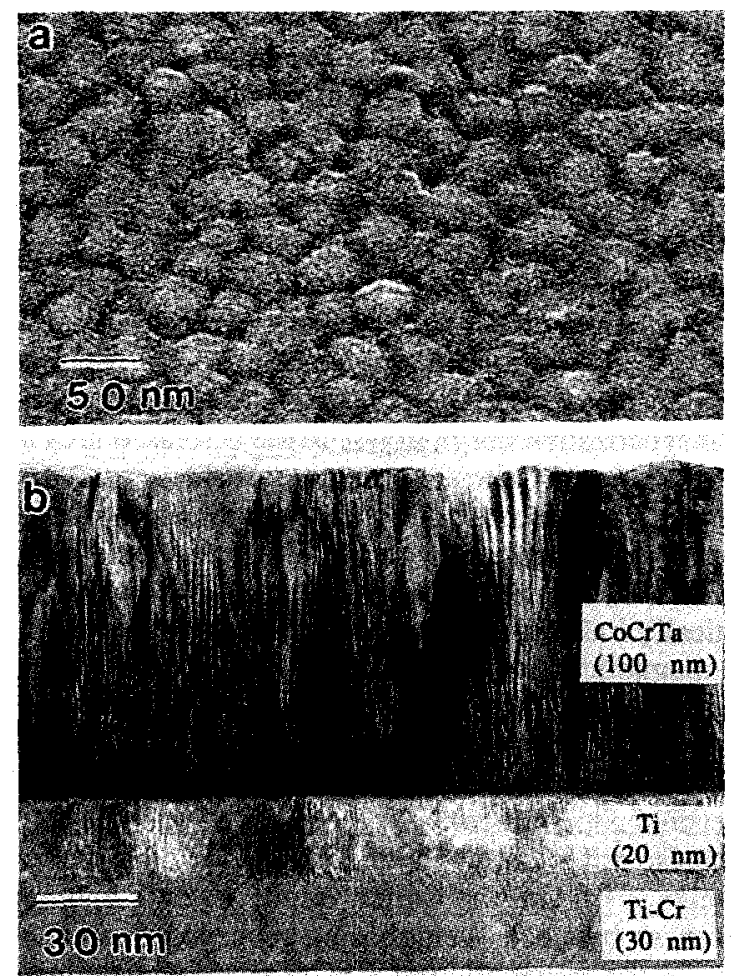

St Substrate

Fig.12 (a)Plane-view SEM and (b)cross-sectional TEM micrographs of $\mathrm{CoCr}_{17} \mathrm{Ta}_{5}$ perpendicular recording medium.

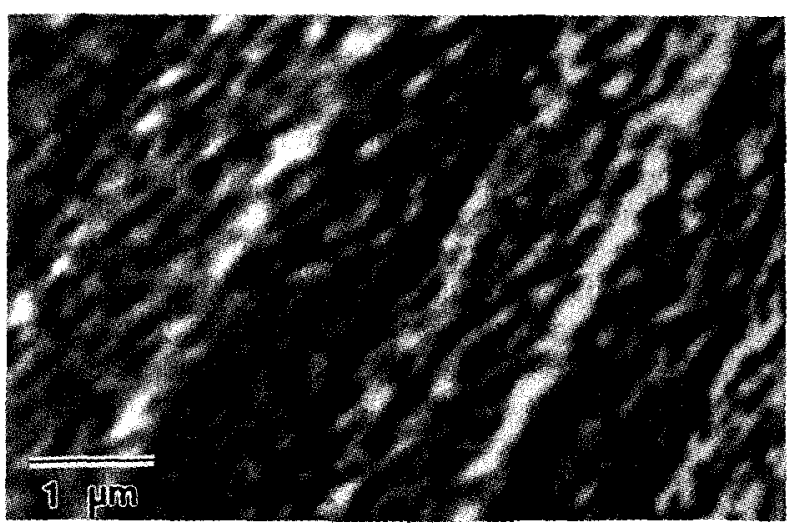

Fig.13 Magnetic force microscope image of $\mathrm{CoCr}_{17} \mathrm{Ta}_{5}$ perpendicular recording medium recorded at $20 \mathrm{kFCI}$.

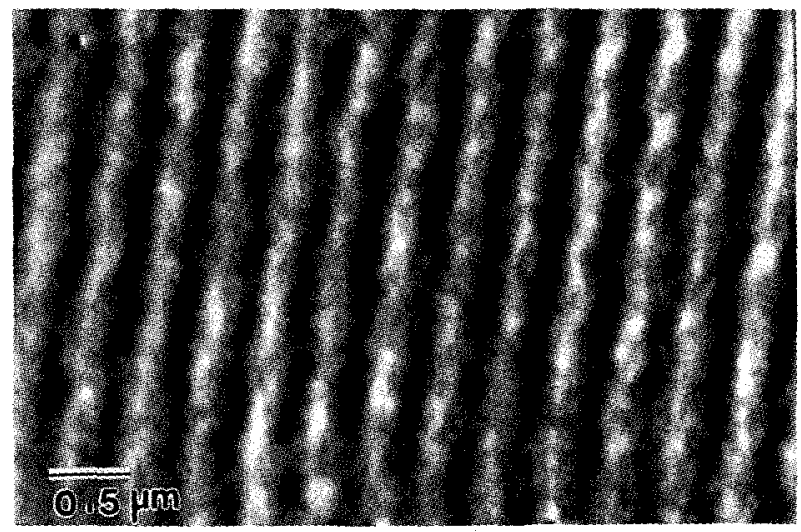

Fig.14 Magnetic force microscope image of $\mathrm{CoCr}_{17} \mathrm{Ta}_{5}$ perpendicular recording medium recorded at $100 \mathrm{kFCl}$.

The MFM image of the sample recorded at $20 \mathrm{kFCI}$, shown in Fig. 13, indicates that there are magnetization irregularities of $0.2-0.35 \mu \mathrm{m}$ within the recorded bit transitions. These irregularities are considered as a result of the local magnetization fluctuations, and/or the reverse magnetic domains whose magnetization directions are either upwards or downwards respective to the film plane[23]. Comparison between the size of crystalline grain diameter and the shape of the magnetization irregularities suggests that each magnetic domain consists of several to a few dozens of crystal grains. The MFM image of the same sample recorded at $100 \mathrm{kFCI}$ ( Fig.14) indicates that, in contrast to the case of longitudinal recording media, the shape of the magnetization transitions is straight and the width, which is smaller than $0.1 \mu \mathrm{m}$, appears to remain unchanged. However, the bit transitions at $100 \mathrm{kFCI}$ recording become discontinuous at some regions due to the presence of reverse magnetic domains. The magnetization structure observation clearly indicates that the major source of medium noise in the $\mathrm{CoCr}_{17} \mathrm{Ta}_{4}$ perpendicular recording medium depends on the presence of magnetic domains of which magnetization directions are probably reversed between the neighboring magnetic domains. It is apparently necessary to decrease the size of magnetic domains well below the recording bit length, by decoupling the magnetic interaction between the magnetic crystal grains and by decreasing the crystal grain diameter. 


\section{OBLIQUELY EVAPORATED RECORDING MEDIUM}

Figure 15 shows a cross-sectional TEM micrograph of the obliquely evaporated $\mathrm{Co}-\mathrm{CoO}$ film. This film consists of h.c.p. $\mathrm{Co}$ and $\mathrm{CoO}$ fine crystal grains of about $5 \mathrm{~nm}$ in diameter which form columnar structures[14]. The easy magnetization axis of this sample is about 30 degrees from the normal to the film plane. MFM images of this sample recorded at 10 and $100 \mathrm{kFCI}$ are shown in Figs.16 and 17, respectively. Well defined bright and dark bands

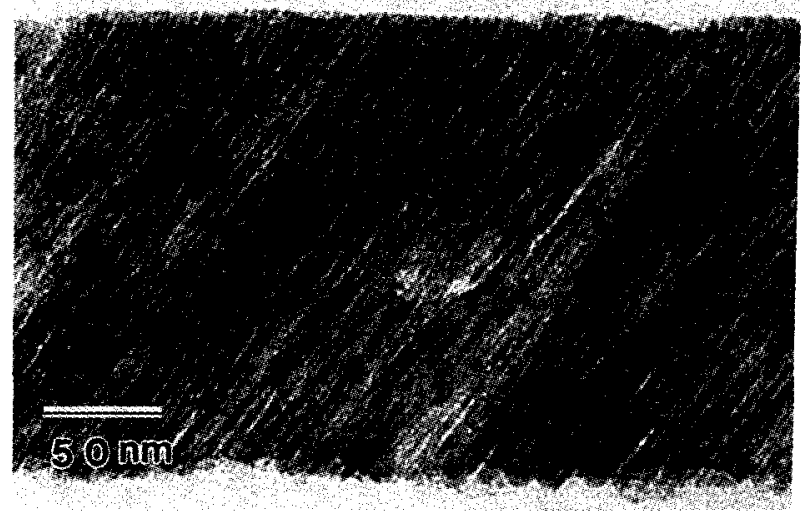

Fig.15 Cross-sectional TEM micrograph of Co-CoO obliquely evaporated medium.

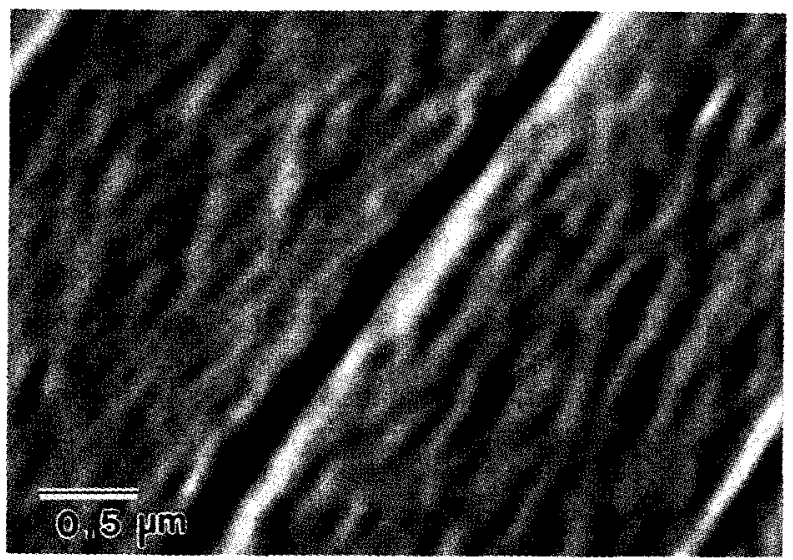

Fig.16 Magnetic force microscope image of $\mathrm{Co}-\mathrm{CoO}$ obliquely evaporated medium recorded at $10 \mathrm{kFCl}$.

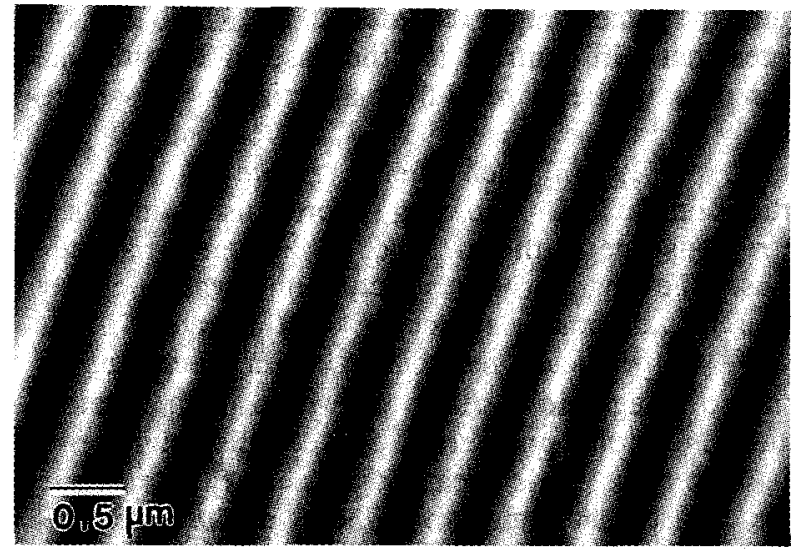

Fig.17 Magnetic force microscope image of $\mathrm{Co}-\mathrm{CoO}$ obliquely evaporated medium recorded at $100 \mathrm{kFCl}$. in these MFM images suggest that sharp magnetization transitions are formed between the neighboring recorded bits whose magnetization directions are oriented in the opposite directions. Such sharp magnetization transitions are probably due to the quasi-perpendicular magnetization which is $\mathbf{3 0}$ degrees inclined from the normal to the film plane. The magnetization irregularities of around 0.1 . $0.25 \mu \mathrm{m}$ are seen within the range of $10 \mathrm{kFCI}$ bit transitions[24]. At a recording density of $100 \mathrm{kFCI}$, the average size of the magnetization irregularities seems to have decreased below $0.1 \mu \mathrm{m}$. The fact that the magnetization irregularities decreases as the recording density increase is clearly correlated to the noise characteristics of this medium. As shown in Fig.2, the medium noise decreased as the linear density increased. Regions of dark contrast of about $0.1 \mu \mathrm{m}$ in diameter observed in the MFM image of Fig.17 are considered to be reverse magnetic domains. The small scale of the magnetization irregularities in this medium is playing an important role in reducing medium noise and improving the linear density characteristics[15].

\section{DISCUSSION}

Medium noise originates from irregularities in recorded bit transitions, local magnetization fluctuations which exist over the recorded area, irregularities at the recorded track edges, etc. Here, the relationships between the first two noise sources and the recorded magnetization structures will be compared for different types of media. The possibilities of further decreasing the media noise will be discussed as well.

For the longitudinal recording media of $\mathrm{CoCrPt} / \mathrm{CoCrPtSi}$, and $\mathrm{CoCrTa}$ films, magnetic interference between the neighboring bit transitions is clearly observed. The magnetic interference deforms the shape of magnetization transitions and broadens the transition width causing the medium noise to increase. This interference is related to the increase of longitudinal recording medium noise when the linear recording density increase. The threshold linear density, a point where the medium noise increases sharply, seems to be related to the size of magnetization irregularities in the dc erased area and in area within the bit transitions of low frequency recordings. The magnetization irregularities are probably due to the presence of magnetic clusters. Each cluster consists of multiple magnetic crystal grains that are magnetically linked. As the bit length approaches the size of the magnetic cluster with the increase of the linear recording density, there will probably be a magnetic interference between two magnetic clusters, belonging to the neighboring recorded bits in order to elongate and form a longer magnetic cluster. The chance of such coupling will increase as the bit length decreases, yielding more bit cross-talks. On the other hand, such magnetic interferences between neighboring recorded bits are not obvious in the perpendicular CoCrTa and the obliquely evaporated $\mathrm{Co}-\mathrm{CoO}$ recording media. There is no drastic increase in the media noise when the linear recording density is increased. This is basically due to the difference in the recording mode. Magnetization irregularities existing within the bit transitions are considered to be the major 
noise source for these media. The advantage of the absence of magnetic interference in perpendicular and obliquely evaporated media is proven experimentally. It helps in achieving high linear density magnetic recording where the media noise reduction becomes very important. However, the size of magnetization irregularities observed especially for the perpendicular $\mathrm{CoCrTa}$ medium seems to be too large, in comparison to that of longitudinal $\mathrm{CoCr}_{16} \mathrm{Ta}_{4}$ medium, to be used for ultra-high density magnetic recording. The medium microstructure needs to be further improved to be used with ultra-high density magnetic recording.

In order to drastically improve the recording characteristics, the size of the magnetization irregularities should be decreased below the minimum bit length of magnetic recording. This applies not only for longitudinal recording media but also for perpendicular and obliquely evaporated recording media. When the size of magnetization irregularities is greater than the bit length, even with a perpendicular or an obliquely evaporated medium, the bit transitions become discontinuous due to the presence of reverse domains whose size is larger than the bit length. In this case, the intensity of the reproduced signal in a read head will decrease, thus degrading the signal to the noise ratio. In Table 2, the average size of the magnetization irregularities and the magnetic crystal grain diameter are compared in respect to longitudinal $\mathrm{CoCrPt} / \mathrm{CoCrPtSi}$, CoCrTa media, the perpendicular $\mathrm{CoCrTa}$ medium, and the obliquely evaporated $\mathrm{Co}-\mathrm{CoO}$ medium. The size of the magnetization irregularities for these samples is more than several times greater than the size of magnetic crystal grains. These results indicate that a unit of magnetization irregularity, or in other words, a magnetic cluster, consists of roughly $40\left(\mathrm{CoCr}_{16} \mathrm{Ta}_{4}\right.$ longitudinal recording medium) to $580(\mathrm{Co}-\mathrm{CoO}$ obliquely evaporated medium) magnetic crystals. It may be possible to decrease this unit size by decreasing the crystal grain diameter together with enhancing the magnetic separation between the neighboring magnetic crystal grains. Enhancing the magnetic separation will result in the decrease in the number of crystal grains in a magnetic cluster. As observed for longitudinal CoCrTa media, the increase in the nonmagnetic $\mathrm{Cr}$ concentration of the magnetic thin film is very effective in reducing the magnetic interaction between crystal grains due to enhanced segregation of $\mathrm{Cr}$ at grain boundaries. The media fabrication process and the media composition have to be further improved to yield microstructures that are suitable for higher density magnetic recording.

Table 2 Comparison of magnetic crystal grain diameter and size of magnetization irregularities for differnt types of thin film media.

\begin{tabular}{|c|c|c|c|c|c|}
\hline & \multicolumn{3}{|c|}{ Longitudinal } & \multirow{2}{*}{\begin{tabular}{|l|} 
Perpendicular \\
CoCriTTa5
\end{tabular}} & \multirow{2}{*}{\begin{tabular}{|c|} 
Oblique \\
Co-CoO
\end{tabular}} \\
\hline & $\mathrm{CoCrP} / \mathrm{CoC} r \mathrm{PtSi}$ & Cocriotad & oCr16Ta4 & & \\
\hline $\begin{array}{c}\text { Grain diameter } \\
\mathrm{d}_{\mathrm{g}} \mathrm{nm}\end{array}$ & 25 & 20 & 20 & 35 & 5 \\
\hline $\begin{array}{l}\text { Size of } \\
\text { magntization } \\
\text { irregularities } \\
d_{m} \mathrm{~nm}\end{array}$ & 200 & 160 & 120 & 250 & 120 \\
\hline$\frac{d_{m}}{d_{g}}$ & 8.0 & 8.0 & 6.0 & 7.1 & 24 \\
\hline
\end{tabular}

\section{CONCLUSIONS}

The physical microstructures and recorded magnetization microstructures of Co-based longitudinal, perpendicular, and obliquely evaporated recording media are investigated and related to the media noise characteristics. The following results are obtained.

(1) Two types of magnetization irregularities are observed. One is associated with the magnetization transitions and the other associates with the magnetization fluctuations existing all over the recorded region. The former type of magnetization irregularity is observed for longitudinal recording media and the latter type is observed in all the thin film media.

(2) For the longitudinal recording media, the magnetic interference between the neighboring recorded bits is likely to be the major factor in noise augmentation when the linear recording density is increased

(3) For perpendicular and obliquely evaporated recording media, the magnetization irregularities, existing all over the recorded region and in the dc erased area, are the major source of media noise.

(4) The magnetization irregularities indicates the presence of magnetic clusters which consist of magnetically linked crystal grains.

(5) The size of the magnetization irregularities is more than several times greater than the average diameter of the magnetic crystal grains.

(6) In order to improve the noise and linear recording density characteristics, it is necessary to reduce the size of the magnetization irregularity below the minimum bit length of magnetic recording regardless of the medium type.

\section{ACKNOWLEDGEMENTS}

The authors are grateful to Dr. K. Koike, Dr. H. Matsuyama of Advanced Research Laboratory, Hitachi Ltd., Dr. N. Inaba, Mr. T. Takayama, Mr. Y. Matsuda, Mr. M. Suzuki, Mr. Y. Hirayama, and Mr. F. Tomiyama of Hitachi Central research Laboratory for their contributions on the experiments and discussions. Special thanks are due to Dr. Y. Sugita for his encouragements and useful discussions.

\section{REREFENCES}

[1] T. Chen and T. Yamashita, IEEE Trans. Magn. $24,2700(1988)$.

[2] J. -G. Zhu and H. N. Bertram, IEEE Trans. Magn. 24, 2706 (1988).

[3] Y. Matsuda, M. Inaba, M. Suzuki, H. Takano, and M. Futamoto, J. Mag. Soc. Jpn. 15 Suppl. S2 1001(1993).

[4] H. Aoi, F. Tomiyama, Y. Shiroishi, K. Yoshida, and Y. Sugita, IEEE Trans. Magn. 29, 3715(1993).

[5] M. F. Doerner, T. Yogi, D. S. Parker, S. Lambert, B. Hermsmeier, O. C. Allegranza, and T. Nguyen, IEEE Trans. Magn. 29, 3667(1993).

[6] K. E. Johnson, K. J. Schulz, and J. M. Severson, IEEE Trans. Mahn. 29, 3670(1993).

[7] T. Yogi, C. Tsang, T. A. Nguyan, K. Ju, G. L. Gorman, and G. Gastillo, IEEE Trans. Magn. 26, 2271(1990). 
[8] M. Futamoto, F. Kugiya, M. Suzuki, H. Takano, Y. Matsuda, N. Inaba, Y. Miyamura, K. Akagi, T. Nakao, S. Sawaguchi, T. Munemoto, and T. Takagaki, IEEE Trans. Magn. 27, 5280(1991).

[9] S. Yamamoto, Y. Nakamura, and S. Iwasaki, IEEE Trans. Magn. 23, 2070(1987).

[10] Y. Honda, M. Futamoto, S. Hasegawa, T. Kawasaki, F. Kugiya, M. Koizumi, K. Yoshida, and A. Tonomura, J. de Physique Colloque C8, Tome 49, 1969(1988).

[11] K. Sato, H. Wakamatsu, and M. Shinohara, IEEE Trans. Magn. 29, 3730(1993).

[12] H. Muraoka and Y. Nakamura, J. Mag. Soc. Jpn. 17, Suppl. S2, 222(1993).

[13] Y. Matsuda, M. Suzuki, Y. Hirayama, Y. Honda and M. Futamoto, Proc. PMRC'94(11pA-12).

[14] T. Takayama and K. Yoshida, J. Magn. Soc. Jpn. 13 Suppl. S1, 819(1989).

[15] T. Takayama, K. Yoshida, and Y. Honda, Technical Report of ICICE, MR94-1(1994-05).

[16] T. Takayama, K. Yoshida, Y. Ogawa, A. Yano, and O.

Kitakami, Proc. 6th Int. Conf. Ferrites(ICF 6, Tokyo, 1992) pp 1440.

[17] N. Inaba, Y. Matsuda, M. Suzuki, A. Nakamura, and M. Futamoto, J. Appl. Phys. 75, 6126(1994).

[18] Y. Honda, N. Inaba, M. Suzuki, A. Kikugawa, and M. Futamoto, IEEE Trans. Magn. 29, 3721(1993).

[19] H. Matsuyama and K. Koike, J. Electron Microscopy 43, 157(1994).

[20] Y. Honda, M. Suzuki, F. Tomiyama, H. Matsuyama, N. Inaba, K. Koike, and M. Futamoto, Jpn. J. Appl. Phys.33, L1083(1994).

[21] H. Matsuyama, K. Koike, F. Tomiyama, Y. Shiroishi, A. Ishikawa, and H. Aoi, IEEE Trans. Magn. 30(1994), to be published.

[22] M. Futamoto, Y. Honda, N. Inaba, F. Tomiyama, Y. Hosoe, Y. Shiroishi, Y. Yajima, and H. Matsuyama, MMMIntermag Conference'94 (Paper AA-03).

N. Inaba et al. to be submitted.

[23] K. Ouchi, J. Mag. Soc. Jpn., 13 Suppl. S1, 611(1989).

[24] H. Matsuyama, K. Koike, and T. Takayama, Abstract of 18th Jpn. Magn. Soc. Conf.(1994, Sept.). 